\title{
The Use of Biomarkers for the Early Detection of Vulnerable Atherosclerotic Plaques and Vulnerable Patients. A Review
}

\author{
Theodora Benedek ${ }^{1}$, Pál Maurovich-Horváth², Péter Ferdinandy ${ }^{3,4}$, Béla Merkely \\ ${ }^{1}$ Department of Cardiology, University of Medicine and Pharmacy, Tîrgu Mureş, Romania \\ ${ }^{2}$ MTA-SE Cardiovascular Imaging Research Group, Heart and Vascular Center, Semmelweis University, Budapest, Hungary \\ ${ }^{3}$ Pharmahungary Group, Szeged, Hungary \\ 4 Department of Pharmacology and Pharmacotherapy, Semmelweis University, Budapest, Hungary
}

\begin{abstract}
Acute coronary syndromes represent the most severe consequences of atherosclerosis, most often triggered by the rupture of a coronary plaque, which, for various reasons, has become unstable. In many cases, these rupture-prone vulnerable plaques are difficult to diagnose, because they do not always cause significant obstruction noticeable by coronary angiography. Therefore, new methods and tools for the identification of vulnerable plaques have been proposed, many of which are currently under study. Various biomarkers have been suggested as predictors of a vulnerable plaque, as well as indicators of an increased inflammatory status associated with higher patient susceptibility for plaque rupture. Integration of such biomarkers into multiple biomarker platforms has been suggested to identify superior diagnostic algorithms for the early detection of the high-risk condition associated with an unstable plaque. The aim of this review is to summarize recent research related to biomarkers used for the early detection of vulnerable plaques and vulnerable patients.
\end{abstract}

Keywords: vulnerable plaque, vulnerable patient, FFR-CT, atheromatosis

\section{ARTICLE HISTORY}

Received: 27 June, 2016

Accepted: 3 September, 2016

\section{CORRESPONDENCE}

Pál Maurovich-Horváth

Városmajor u. 68

1122 Budapest, Hungary

Tel: +3614586847

E-mail: maurovich.horvat@gmail.com

\section{BACKGROUND}

Cardiovascular diseases (CVD) are amongst the leading causes of mortality and morbidity worldwide. ${ }^{1}$ According to the World Health Organization (WHO), 17.5 million people died from CVD in 2012, representing 31\% of all global deaths. The majority of $\mathrm{CV}$ deaths are caused by the progression of atherosclerosis, resulting in acute coronary syndromes or stroke. WHO also estimates that 7.4 million deaths occurred due to coronary heart disease and 6.7 million were caused by stroke, and it is currently predicted that 23.6 million people will die annually by 2030 due to CVD. ${ }^{1}$

Acute myocardial infarction is a disease with a strong effect on patients' health and quality of life. If not treated promptly by appropriate reperfusion therapy, severe complications, such as heart failure, arrhythmia, thromboembolism or even death, may occur. Myocardial infarction (MI) can have a substantial negative impact on the 
social, economic and psychological status of the patients, who prior to the MI were active. ${ }^{2}$ If the patient is treated in time, and a coronary stent is implanted at the site of the infarction, there is a lower risk of complications. However, limitations in exercise capacity, with consequent deterioration of the quality of life will remain. Therefore, effective preventive strategies aimed at moderating the progression of the "vulnerable patients" towards developing an acute myocardial infarction could lead not only to a significant reducing in healthcare costs, but also to a substantial improvement in the quality of life in these patients. ${ }^{3}$

Acute coronary syndromes (ACS) represent the most severe consequences of atherosclerosis, most often being triggered by the rupture of a coronary plaque, which, for different reasons, becomes unstable. Plaque rupture has been identified as the pathophysiologic substrate in more than $75 \%$ of all fatal heart attacks. ${ }^{4}$ However, it has been proved that $25-40 \%$ of STEMI are produced by plaques with an intact fibrous cap. These types of plaques usually present with lower degrees of lipid reach core, and less frequently have a thin-capped fibroatheroma. ${ }^{5}$ One major question is why a coronary plaque suddenly becomes unstable and ruptures. Timely identification of ruptureprone plaques could lead to the appropriate initiation of suitable therapeutic measures. The ability to detect the risk of having a myocardial infarction, and to contribute to the proper treatment of these lesions before plaque rupture and consequent myocardial infarction, would assist in preventing plaque rupture and the subsequent acute coronary event. ${ }^{6}$ In many cases, vulnerable plaques are difficult to diagnose, because they do not always cause significant obstruction as seen by coronary angiography. 4

Standard diagnostic tools, such as conventional coronary angiography, focus more on luminal narrowing than on plaque characteristics and are unable to estimate the vulnerability of a coronary plaque, and therefore cannot predict if a plaque is prone to rupture with the subsequent risk of an acute coronary event. ${ }^{4}$

Various biomarkers have been proposed to characterize vulnerable plaques and to gauge the increased inflammatory status associated with patient vulnerability. Integration of such biomarkers into multiple biomarker platforms has been suggested with the aim of achieving superior diagnostic algorithms for the early detection of the high-risk conditions. In this review, the most recent progress relating to the use of biomarkers for the early detection of vulnerable plaques and vulnerable patient, is summarized.

\section{VULNERABLE PLAQUE AND VULNERABLE PATIENT}

The concept of the vulnerable patient has been introduced to describe a status characterized by an increased susceptibility to the localized action of factors destabilizing the plaque, and exposing the person to a higher risk of plaque rupture. Nowadays it is considered that an acute coronary event is the result of an interaction between plaque vulnerability and patient vulnerability. ${ }^{7}$ Therefore, the current approach for predicting the risk of an acute coronary event is based on the identification of both vulnerable coronary plaques and the identification of biomarkers characterizing the so-called "vulnerable patient".

Recently, the concept of plaque sealing has been introduced as a new therapeutic approach consisting of the implantation of a bio-absorbable scaffold at the site of a vulnerable plaque to prevent plaque rupture. ${ }^{8}$ It is wellknown that a large number of vulnerable coronary plaques do not result in any significant stenosis, making the decision to stent or not, very difficult in clinical practice. ${ }^{9} \mathrm{Cur}-$ rently, there is no consensus either when this technique should be employed in the treatment of unstable coronary plaques, or the role of different biomarkers as indicators of a specific therapeutic strategy.

\section{SERUM BIOMARKERS CHARACTERIZING THE VULNERABLE PATIENT}

Serum biomarkers expressing an increased inflammatory status or disease severity, such as hsC-reactive protein, cardiac enzymes - troponin ( $\mathrm{Tn}$ ) and its highly sensitive form (hsTn), myoglobin, C-reactive protein (CRP) and its highly sensitive form (hsCRP), brain natriuretic peptide and NT-proBNP, galectine-3, neutrophil gelatinaseassociated lipocalin, myeloperoxidase (MPO), cytokines (Growth differentiation factor 15) or interleukines (Soluble ST2), have been traditionally associated with an increased risk of an acute coronary event. As such, their detection could play an important prognostic role, though this role is still under investigation. Other novel serum biomarkers that are involved in the vulnerabilization process of the coronary atheromatous plaque include the matrix metalloproteinases (MMPs), the oxidized LDL cholesterol, apolipoprotein B, as well as the homocysteine levels. ${ }^{10-14}$

Micro-RNAs are RNAs synthesized in the nucleus that regulate gene expression, and play a significant role in ischemic cardiovascular diseases. Recently it has been proposed that they can serve as biomarkers to monitor progression after an acute myocardial infarction, heart 
failure and coronary artery disease, as miRNA diseasespecific signatures have been identified in a variety of $\mathrm{CV}$ diseases. For instance, miR-1, miR-133a, miR-133b, miR208a and miR-499 have been demonstrated to be associated with acute myocardial infarction. ${ }^{15}$ However, the exact panel of microRNA specific for vulnerable plaque has not been identified so far, and it would be interesting to elucidate if these miRNA reflect the vulnerability of a plaque or the vulnerability of a patient.

\section{LOCALLY RELEASED BIOMARKERS AT THE SITE OF THE VULNERABLE PLAQUE}

Impaired function of the coronary endothelium leads to local release into the coronary circulation of inflammatory or pro-thrombotic biomarkers. ${ }^{16}$ So far, most biomarker studies have focused on circulating biomarkers released from plaques throughout the entire circulation, not just those locally sampled within the coronary arteries. The association between the various locally released biomarkers expressing inflammation, endothelial dysfunction, and the risk for plaque rupture has not been elucidated so far. ${ }^{17}$ Likewise, the role of different biomarkers derived from the coronary circulation, as an expression of increased inflammation associated with vulnerable plaques, has not been clearly demonstrated. ${ }^{18}$ Currently, locally released biomarkers at the site of the vulnerable plaque, expressing inflammation and endothelial dysfunction, can be determined using liquid biopsy techniques. ${ }^{19}$ This biopsy system was developed recently in order to provide a better indication of the risk associated with increased levels of biomarkers collected along an atheromatous plaque, based on the calculation of the biomarker gradient between the region located upstream and downstream of the diseased tissue. The ongoing clinical trial "Study to Detect Biomarker Gradients in Coronary Arteries Using the Liquid Biopsy System (CS1)" will investigate this new procedure, aiming to enroll 70 patients with coronary artery disease undergoing routine percutaneous coronary intervention (PCI). The study will test the feasibility of the new liquid biopsy system device for the detection of local biomarkers released by diseased coronary arteries, and will also assess the link between these locally released biomarkers and the cardiovascular risk of the patient.

\section{IMAGING-DERIVED BIOMARKERS CHARACTERIZING VULNERABLE PLAQUE}

Despite recent advances in the characterization of multiple imaging biomarkers associated with plaque vulnerability, there is no clear consensus on the predictive value of these biomarkers for predicting an acute coronary event. ${ }^{20}$

A vulnerable plaque is usually characterized by a large necrotic core, a thin fibrous cap with macrophage infiltration within the cap, a large lipid pool, and the presence of several specific signs such as positive remodeling, irregular calcifications, and low attenuation at cardiac computed tomography. ${ }^{4,21}$ When these characteristics are present, the fibrous cap may rupture, and the lipid core, which is thrombogenic, is exposed to the blood flow, inducing thrombus formation and causing an acute coronary syndrome. ${ }^{22,23}$ At the same time, new imaging biomarkers such as the epicardial fat thickness (EFT) have been demonstrated to be associated with coronary artery disease. However the association of EFT with vulnerable plaques or with patient vulnerability is still unclear, and controversies still exist regarding the role of several characteristics of EFT such as volume or spatial distribution around coronary arteries, in predicting associated cardiovascular risk. $^{24-27}$

The morphological characteristics associated with unstable plaques are evaluated by three main imaging methods: Coronary Computed Tomography Angiography (CCTA), Intravascular ultrasound with virtual histology (VH-IVUS), and Optical Coherence Tomography (OCT). The most common image-derived biomarkers associated with plaque vulnerability, as identified by leading imaging technologies, are summarized in Table 1.

Different studies have suggested an association between various parameters characterizing vulnerable plaques, as identified by one of the three imaging techniques, and development of acute coronary syndromes. ${ }^{28,29}$ However, these studies reported qualitative data related only to one imaging method, without taking into consideration the global picture of the vulnerable plaque. This could possibly be better characterized by the summation of all the information obtained by these techniques. ${ }^{24}$

a) Coronary Computed Tomography Angiography for the assessment of vulnerable plaques. CCTA is a noninvasive technique able to identify specific parameters associated with plaque vulnerability, such as spotty calcifications, active remodeling, and burden with a low-density plaque. Available software makes it possible to use this technique for quantitative analysis of plaque components based on different $\mathrm{CT}$ attenuations within the plaque. ${ }^{30}$

Motoyama et al. found that the presence of three markers of instability in the same plaque, spotty calcification, active remodeling and low-density atheroma, identified by cardiac computed tomography, were associated with the development of an acute coronary syndrome at two years. ${ }^{31}$ 
TABLE 1. Image-derived biomarkers associated with plaque vulnerability, as identified by main imaging technologies

\begin{tabular}{lcccc}
\hline $\begin{array}{l}\text { Image-derived biomarkers for plaque } \\
\text { vulnerability }\end{array}$ & CT & VH-IVUS & OCT & NIRF \\
\hline Necrotic core & NO & YES & NO & NO \\
Positive remodelling & YES & YES & NO & NO \\
Spotty calcification & YES & YES & NO & NO \\
Napkin-ring sign & YES & NO & NO & NO \\
Lipid-reach plaque & YES & YES & YES & YES \\
Plaque erosion & NO & NO & YES & NO \\
Thin cap fibroatheroma & NO & NO & YES & NO \\
Thrombus & NO & NO & YES & NO \\
Macrophage content & NO & NO & YES & NO \\
\hline
\end{tabular}

CCTA - Coronary Computed Tomography Angiography; VH-IVUS - Virtual histology-intravascular ultrasound; OCT - optical coherence tomography; NIRS - near infrared spectroscopy

Pal Maurovich-Horvat et al. described the presence of a ring-like attenuation pattern, also known as the napkin-ring sign, of the coronary atherosclerotic vulnerable plaques, showed in coronary computed tomography angiography (CCTA). ${ }^{32}$ Napkin-ring sign has been identified as a characteristic feature of the vulnerable plaque, consisting in the presence of a low CT attenuation in the center of the plaque close to the lumen, surrounded by a rim area of a high attenuation. The specificity of this sign to identify unstable plaques was $98.9 \%$ (95\% CI: 97.6-100\%).33 In this study, investigating twenty-one coronary arteries from seven donor hearts and correlating the histological features with CCTA appearances, the presence of the napkin-ring sign was a strong predictor for advanced coronary lesions. The study emphasized the ability of CCTA to recognize advanced atherosclerotic lesions associated with high vulnerability and increased probability for adverse cardiovascular events. ${ }^{32}$

In a previous study, it was shown that unstable plaques are characterized by larger volumes of low-density lipid cores, and a critical volume of $0.2 \mathrm{ml}$ very low CT density plaque ( $<30 \mathrm{HU}$ ) was associated with the development of an acute coronary syndrome by Benedek et al. ${ }^{34}$

New CT-based imaging biomarkers, such as epicardial fat thickness, have been proposed as markers of coronary artery disease and the severity of plaque instability. A recent publication described the role of EFT in characterizing the severity of coronary artery disease. ${ }^{24}$ However, the role of this EFT as a biomarker describing patient vulnerability and expressing an increased inflammatory status in patients with vulnerable plaques is yet to be clarified. At the same time, several new hypotheses regarding the role of new CT-based biomarkers have been proposed and are currently under investigation, such as the role of trans- stenotic contrast density gradient in predicting the plaque severity and vulnerability. ${ }^{34}$

b) Optical coherence tomography (OCT) is an intracoronary imaging technology which provides accurate data related to plaque morphology and quantification, and is considered to be the gold standard for measurements of the fibrous cap thickness and visualization of intracoronary thrombus or intimal rupture. The most frequently used OCT-derived biomarker for the assessment of plaque vulnerability is the thickness of the fibrous cap, and OCT studies have identified the presence of a thin fibrous cap, less than 65 microns, as a high-risk indicator of plaque rupture. ${ }^{28}$ New OCT-based biomarkers characterizing vulnerable plaques have been proposed, such as the accumulation of macrophages in the fibrous cap, and these can be detected easily with OCT. ${ }^{28}$

Several OCT studies have demonstrated the presence of lipid-rich plaques in $100 \%$ of ruptured plaques. However, this biomarker was present in only $43 \%$ of plaques with an intact fibroatheroma cap. ${ }^{5}$

In an OCT substudy of the TOTAL trial (ThrOmbecTomy versus PCI Alone), the lipid content was lower in culprit plaques with intact fibrous plaques as compared to ruptured plaques. However, a significant lipid core was present in all the culprit plaques, while the thickness of the fibrous cap was significantly lower in ruptured plaques as compared to intact cap plaques $(62.05 \pm 9.13$ microM vs. $91.03 \pm 16.97$ microM, $\mathrm{p}<0.0001) .{ }^{5}$

c) VH-IVUS can combine intracoronary imaging data with a color coded representation of plaque components, classifying them as fibrous, calcific, lipid reach or necrotic core, at the same time quantifying plaque components and volumes. ${ }^{35}$ However, it does not provide a clear assessment of the fibrous cap thickness or intracoronary thrombus. ${ }^{36}$ 
IVUS studies identified the presence of a large necrotic core as a marker of vulnerability. ${ }^{37}$ A previous study described the association between a necrotic core determined by $\mathrm{VH}-$ IVUS and the low-density core within the unstable coronary plaque, demonstrating that the dark spots associated with the napkin-ring sign shown by CT, represent in fact the necrotic core of the vulnerable plaque. ${ }^{38}$

d) Near infrared spectroscopy is a technique used for evaluation of the lipid content of the atheromatous plaque, as a direct indicator of its vulnerability. It provides the advantage of precise identification of lipid-rich plaques, providing a chemogram of the arterial wall. ${ }^{29}$

Serial Radiofrequency-IVUS and Near Infrared Spectroscopy proved to be efficient techniques for monitoring the size and regression of the necrotic core, as demonstrated in the Integrated Biomarker and Imaging Study 3 (IBIS-3). ${ }^{39}$

\section{NEW ADVANCES IN COMPLEX BIOMARKER DETECTION IN VULNERABLE PLAQUES}

Coronary shear stress is a new biomarker characterizing coronary circulation. Determination of coronary shear stress has become possible due to advanced techniques of computational fluid dynamics, which are based on the computerized processing of CT or OCT derived image sequences. ${ }^{26,40}$ It has been recognized that unstable plaques develop particularly in sites with a low shear stress, and that shear stress directly relates to the risk of developing an acute coronary event. ${ }^{41}$ It is also well-known that the local impaired function of the coronary endothelium, caused by the presence of a large low-density core, may indicate plaque vulnerability, and that shear stress is a direct indicator of local impaired function of the coronary endothelium..$^{42}$ Yi Wang et al. stated that a high shear stress is associated with vulnerable plaque formation and also with vascular remodeling. ${ }^{43}$ High shear stress might play a role in the expression of the vascular endothelial growth factor, which induces angiogenesis and also creates lesions in the vascular barrier function. ${ }^{44}$ However, it is still unclear if high shear stress is directly related to the rupture of a vulnerable plaque.

Noninvasive determination of coronary flow reserve using CT image datasets and computational flow dynamics has been shown to represent an alternative to invasive FFR in determining the functional significance of a coronary plaque. 45

The noninvasive measurement of FFR combines anatomical assessment of lesion severity provided by CT with the hemodynamic assessment of functional significance, provided by computer modeling. ${ }^{46}$ In a study which com- bined the diagnostic value of CT imaging of coronary plaques with noninvasive FFR for the identification of lesions causing ischemia, the CT markers of plaque vulnerability such as active remodeling, spotty calcification and low attenuation plaque were associated with ischemia, and improved the identification and reclassification of coronary lesions causing ischemia, over stenosis degree evaluated with CT plus FFR, measured using computational fluid dynamics applied to CT images. ${ }^{47}$ However, the application of this technique in generating image-based biomarkers characterizing vulnerable plaques is still under investigation. In a recent study, Ahmadi A et al. stated that abnormal FFR could translate lesions that consist of a large necrotic core, with severe ischemia. ${ }^{48}$ Also, they concluded that FFR represents a dependable technique, able to identify a vulnerable coronary plaque independent of luminal stenosis..$^{48}$

Molecular imaging of vulnerable plaque: molecular imaging is a promising field, and many imaging-based biomarkers have been proposed as identifiers of vulnerable plaques. For instance, 18F-labelled nanobodies targeting vascular adhesion molecule-1 were used for PET/ CT imaging of atherosclerotic plaques with a high degree of inflammation inside the plaque, providing relevant information to characterize atherosclerotic plaque inflammation and vulnerability. 49

Nanoparticle-based imaging of vulnerable plaque: when a plaque ruptures, the fibrous cap is injured, and the lipid core, which is thrombogenic, is exposed to circulation, inducing thrombus formation. ${ }^{50}$ Vulnerable, rupture-prone plaques are often relatively large, have a thin fibrous cap, are associated with expansive remodeling, and have a large number of inflammatory cells including macrophage-derived foam cells. ${ }^{51,52}$ Atheromaoriented nanotechnologies have been suggested for the visualization of macrophage accumulations within vulnerable plaques, and may represent a marker of plaque instability. ${ }^{53}$ Specific nanoparticles can be used as contrast agents for ultrasound, computed tomography and MRI to detect macrophages, as the macrophage content is directly associated with the degree of inflammation in the vulnerable plaque. ${ }^{54}$ These macrophage-targeting nanoparticles could be constructed from various materials, such as dielectric silica core covered by a thin metallic, gold or silver shell, and characterize a novel nanoparticle-based atherosclerotic plaques identification agent, detectable by $\mathrm{CT}$, and which is phagocytized by macrophages and monocytes. ${ }^{55,56}$ Such a contrast agent is thus able to differentiate between stable and unstable vulnerable plaques. .7, $58^{-1}$ 


\section{CONCLUSION}

The development of novel assays and imaging techniques has resulted in the advancement of biomarker technology, which allows the detection and classification of both vulnerable plaques and vulnerable patients. However, a complex risk-assessment model based on integration of imaging-derived biomarkers and different patient-specific information related to systemic biomarkers, has yet to be perfected.

\section{CONFLICT OF INTEREST}

Nothing to declare.

\section{ACKNOWLEDGEMENT}

This research was supported via the research grant no. 103544/2016, financed by the Romanian Ministry of European Funds, the Romanian Government and the European Union.

\section{REFERENCES}

1. World Health Organisation, Cardiovascular disease (CVDs), 2016. Available from: http://www.who.int/mediacentre/ factsheets/fs317/en/\#

2. Oldridge N, Guyatt G, Jones N, et al. Effects on quality of life with comprehensive rehabilitation after acute myocardial infarction. Am J Cardiol. 1991;67:1084-1089.

3. Fox KA, Birkhead J, Wilcox R, Knight C, Barth J. British Cardiac Society Working Group on the definition of myocardial infarction. Heart. 2004;90:603-609. doi: 10.1136/ hrt.2004.038679.

4. Choy SY, Mintz GS. What have we learned about plaque rupture in acute coronary syndromes? Curr Cardiol Rep. 2010;12:338343. doi: 10.1007/s11886-010-0113-X.

5. Kajander OA, Pinilla-Echeverri N, Jolly SS, et al. Culprit plaque morphology in STEMI - an optical coherence tomography study: insights from the TOTAL-OCT substudy. EuroIntervention. 2016;12:716-723. doi: 10.4244/EIJV12I6A116.

6. Virmani R, Burke AP, Farb A, Kolodgie FD. Pathology of the unstable plaque. J Am Coll Cardiol. 2006;47:C13-C18. doi: 10.1016/j.jacc.2005.10.065.

7. Lafont A. Basic aspects of plaque vulnerability. Heart. 2003;89:1262-1267.

8. Cuesta J, Rivero F, Bastante T, Benedicto A, Diego G, Alfonso F. Sealing a ruptured non-culprit coronary plaque in a patient with acute myocardial infarction with bioresorbable vascular scaffolds. Rev Port Cardiol. 2015;34:213.e1-3. doi: 10.1016/j. repc.2014.08.029.

9. Waxman S, Ishibashi F, Muller JE. Detection and Treatment of Vulnerable Plaques and Vulnerable Patients Novel Approaches to Prevention of Coronary Events. Circulation. 2006;114:23902411. doi: 10.1161/CIRCULATIONAHA.105.540013.
10. Mittal B, Mishra A, Srivastava A, Kumar S, Garg N. Matrix metalloproteinases in coronary artery disease. Adv Clin Chem. 2014;64:1-72.

11. Minamisawa $\mathrm{M}$, Motoki $\mathrm{H}$, Izawa $\mathrm{A}$, et al. Comparison of Inflammatory Biomarkers in Outpatients With Prior Myocardial Infarction. Int Heart J. 2016;57:11-17. doi: 10.1536/ ihj.15-197.

12. Koening W, Karakas M, Zierer A, et al. Oxidized LDL and the Risk of Coronary Heart Disease: Results from the MONICA/ KORA Augsburg Study. Clin Chem. 2011;57:1196-200. doi: 10.1373/clinchem.2011.165134.

13. Chan DC, Watts GF. Apolipoproteins as markers and managers of coronary risk. QJ Med 2006;99:277-287. doi: 10.1093/ qjmed/hclo27.

14. Sreckovic B, Sreckovic VD, Soldatovic I, et al. Homocysteine is a marker for metabolic syndrome and atherosclerosis. Diabetes Metab Syndr. 2016;pii:S1871-4021(16)30200-4. doi: 10.1016/j.dsx.2016.08.026. [Epub ahead of print]

15. Greco S, Zaccagnini G, Voellenkle C, Martelli F. microRNAs in ischaemic cardiovascular diseases. Eur Heart J. 2016;18: E31-E36. doi: http://dx.doi.org/10.1093/eurheartj/suw012.

16. Madjid $M$, Willerson JT. Inflammatory markers in coronary heart disease. Br Med Bull. 2011;100:23-38. doi: 10.1093/bmb/ ldro43.

17. Hadi HA, Carr CS, Al Suwaidi J. Endothelial Dysfunction: Cardiovascular Risk Factors, Therapy, and Outcome. Vasc Health Risk Manag. 2005;1:183-198.

18. Bentzon JF, Otsuka F, Virmani R, Falk E. Mechanisms of plaque formation and rupture. Circ Res. 2014;114:1852-1866. doi: 10.1161/CIRCRESAHA.114.302721.

19. Razzouk L, Fusaro M, Esquitin R. Novel biomarkers for risk stratification and identification of life-threatning cardiovascular disease: troponin and beyond. Curr Cardiol Rev. 2012;8:109-115.

20. Wang J, Balu N, Canton G, Yuan C. Imaging Biomarkers of Cardiovascular Disease. J Magn Reson Imaging. 2010;32:502515. doi: 10.1002/jmri.22266.

21. Andreou I, Antoniadis AP, Shishido K, et al. How do we prevent the vulnerable atherosclerotic plaque from rupturing? Insights from in vivo assessments of plaque, vascular remodeling, and local endothelial shear stress. J Cardiovasc Pharmacol Ther. 2015;20:261-275. doi: 10.1177/1074248414555005.

22. Cordeiro MAS, Lima JAC. Atherosclerotic plaque characterization by multidetector row computed tomography angiography. J Am Coll Cardiol. 2006;47:C40-C47. doi: 10.1016/j.jacc.2005.09.076.

23. Hoffmann U, Moselewski F, Nieman K, et al. Noninvasive assessment of plaque morphology and composition in culprit and stable lesions in acute coronary syndrome and stable lesions in stable angina by multidetector computed tomography. J Am Coll Cardiol. 2006;47:1655-1662. doi: 10.1016/j.jacc.2006.01.041.

24. Hodas R, Pop S, Opincariu D, et al. Correlations between severity of coronary lesions and epicardial fat volume in patients with coronary artery disease - a multislice CT based study. Journal of Interdisciplinary Medicine. 2016;1:71-78. doi: 10.1515/jim-2016-0014.

25. Zhou Y, Wei Y, Wang X, et al. Decreased adiponectin and increased inflammation expression in epicardial adipose tissue in coronary artery disease. Cardiovascular Diabetology. 2011;10:2. 
26. SamadyH, EshtehardiP, McDanielMC, etal.Coronaryarterywall shear stress is associated with progression and transformation of atherosclerotic plaque and arterial remodeling in patients with coronary artery disease. Circulation. 2011;124:779-788. doi: 10.1161/CIRCULATIONAHA.111.021824.

27. Cecchi E, Giglioli C, Valente S, et al. Role of hemodynamic shear stress in cardiovascular disease. Atherosclerosis. 2011;214:249-256. doi: 10.1016/j.atherosclerosis.2010.09.008.

28. Sinclair H, Bourantas C, Bagnall A, Mintz GS, Kunadian V. OCT for identification of Vulnerable Plaque in Acute Coronary Syndrome. JACC Cardiovasc Imaging. 2015;8:198-209. doi: 10.1016/j.jcmg.2014.12.005.

29. Jaguszewski M, Klingenberg R, Landmesser U. Intracoronary Near Infrared Spectroscopy (NIRS) Imaging for Detection of Lipid Content of Coronary Plaques: Current Experience and Future Perspectives. Curr Cardiovasc Imaging Rep. 2013;6:426-430. doi: 10.1007/s12410-013-9224-2.

30. Ferrante G, Presbitero P, Whitbourn R, Barlis P. Current applications of optical coherence tomography for coronary intervention. Int J Cardiol. 2013;165:7-16. doi: 10.1016/j. ijcard.2012.02.013.

31. Motoyama S, Masayoshi S, Harigaya H, et al. Computed tomographic angiography characteristics of atherosclerotic plaques subsequently resulting in acute coronary syndrome. J Am Coll Cardiol. 2009;54:49-57. doi: 10.1016/j. jacc.2009.02.068.

32. Maurovich-Horvat P, Schlett CL, Alkadhi $\mathrm{H}$, et al. The napkin-ring sign indicates advanced atherosclerotic lesions in coronary CT angiography. JACC Cardiovasc Imaging. 2012;5:1243-1252. doi: 10.1016/j.jcmg.2012.03.019.

33. Maurovich-Horvat $P$, Hoffman U, Vorpahl M, Nakano M, Virmani R, Alkadhi H. The Napkin-Ring Sign: CT Signature of High-Risk Coronary Plaques? JACC Cardiovascular Imaging. 2010;3:440-444. doi: 10.1016/j.jcmg.2010.02.003.

34. Benedek T, Gyöngyösi M, Benedek I. Multislice computed tomographic coronary angiography for quantitative assessment of culprit lesions in acute coronary syndromes. Can J Cardiol. 2013;29:364-371. doi: 10.1016/j.cjca.2012.11.004.

35. Batty JA, Subba S, Luke P, Gigi LWC, Sinclair H, Kunadian V. Intracoronary imaging in the detection of vulnerable plaques. Curr Cardiol Rep. 2016;18:28. doi: 10.1007/s11886-016-0705-1.

36. Finn AV, Chandrashekhar Y, Narula J. Vulnerable plaques: from PROSPECT to prospects... JACC Cardiovasc Imaging. 2012;5:334-336. doi: 10.1016/j.jcmg.2012.02.004.

37. Kataoka Y, John JS, Wolski K, et al. Larger lipid pools associate with features of plaque vulnerability on optical coherence tomography. J Am Coll Cardiol. 2013;61(10_S). doi:10.1016/ So735-1097(13)61795-7.

38. Benedek T, Jako B, Benedek I. Plaque Quantification by Coronary CT and Intravascular Ultrasound Identifies a Low CT Density Core as a Marker of Plaque Instability in Acute Coronary Syndromes. Int Heart J. 2014;55:22-28.

39. Oemrawsingh RM, Garcia-Garcia HM, van Geuns RJ, et al. Integrated Biomarker and Imaging Study 3 (IBIS-3) to assess the ability of rosuvastatin to decrease necrotic core in coronary arteries. EuroIntervention. 2016;12:734-739. doi: 10.4244/EIJV12I6A118.

40. Thondapu V, Bourantas CV, Foin N, Jang IK, Serryus PW, Barlis P. Biomechanical stress in coronary atherosclerosis: emerging insights from computational modelling. Eur Hear
J. 2016; pii:ehv689. doi: http://dx.doi.org/10.1093/eurheartj/ ehv689 ehv689. [Epub ahead of print].

41. Wentzel JJ, Chatzizisis YS, Gijesen JH, Giannoglou GD, Feldman $\mathrm{CL}$, Stone PH. Endothelial shear stress in the evolution of coronary atherosclerotic plaque and vascular remodeling: current understanding and remaining questions. Cardiovasc Res. 2012;96:234-243. doi: 10.1093/cvr/cvs217.

42. Koskinas KC, Chatzizisis YS, Baker AB, Edelman ER, Stone PH, Feldman CL. The role of low shear stress in the conversion of atherosclerotic lesions form stable to unstable plaque. Curr Opin Cardiol. 2009;24:580-590. doi: 10.1097/ HCO.obo13e328331630b.

43. Yi Wang, Juhui Qiu, Shisui Luo, et al. High shear stress induces atherosclerotic vulnerable plaque formation through angiogenesis. Regen Biomater. 2016;3:257-267. doi: 10.1093/ $\mathrm{rb} / \mathrm{rbw021}$.

44. Weis SM, Cheresh DA. Pathophysiological consequences of VEGF-induced vascular permeability. Nature. 2005;437:497504. doi: 10.1038/nature03987.

45. Norgaard BL, Leipsic J, Gaur S, et al. Diagnostic Performance of Noninvasive Fractional Flow Reserve Derived from Coronary Computed Tomography Angiography in Suspected Coronary Artery Disease. J Am Coll Cardiol. 2014;63:1145-1155. doi: 10.1016/j.jacc.2013.11.043.

46. Secchi F, Alì M, Faggiano E, et al. Fractional flow reserve based on computed tomography: an overview. Eur Heart J. 2016;18:E49-E56.

47. Nakazato R, Park HB, Gransar H, et al. Additive diagnostic value of atherosclerotic plaque characteristics to non-invasive FFR for identification of lesions causing ischaemia: results from a prospective international multicentre trial. EuroIntervention. 2016;12:473-481. doi: 10.4244/EIJY15M09_02.

48. Ahmadi A, Stone GW, Leipsic J, et al. Association of Coronary Stenosis and Plaque Morphology With Fractional Flow Reserve and Outcomes. JAMA Cardiol. 2016;1:350-357. doi: 10.1001/ jamacardio.2016.0263.

49. Bala G, Blykers A, Xavier C, et al. Targeting of vascular cell adhesion molecule-1 by $18 \mathrm{~F}$-labelled nanobodies for PET/ CT imaging of inflamed atherosclerotic plaques. Eur Heart J Cardiovasc Imaging. 2016;17:1001-1008. doi: 10.1093/ehjci/ jev346.

50. Shah PK. Mechanisms of plaque and rupture. J Am Coll Cardiol. 2003;41:15S-22S.

51. Kolodgie FD, Gold HK, Burke AP, et al. Intraplaque hemorrhage and progression of coronary atheroma. N Engl J Med. 2003;349:2316-2325.

52. Davies MJ. Acute coronary thrombosis: the role of plaque disruption and its initiation and prevention. Eur Heart J. 1995;16:3-7.

53. Tu C, Ng TSC, Sohi H, et al. Receptor targeted Iron Oxide Nanoparticles for Molecular MR imaging of inflamed Atherosclerotic Plaques. Biomaterials. 2011;32:7209-7216. doi: 10.1016/j.biomaterials.2011.06.026.

54. Weissleder R, Nahrendorf M, Pittet MJ. Imaging Macrophages with nanoparticles. Nature Materials. 2014;13:125-138. doi: 10.1038/nmat3780.

55. Cormode DP, Jarzyna PA, Mulder WJM, Fayad ZA. Modified natural nanoparticles as contrast agents for medical imaging. Adv Drug Deliv Rev. 2010;62:329-338. doi: 10.1016/j. addr.2009.11.005. 
56. Cormode DP, Naha PC, Fayad ZA. Nanoparticle Contrast Agents for Computed Tomography: A Focus On Micelles. Contrast Media Mol Imaging. 2014;9:37-52. doi: 10.1002/cmmi.1551.

57. von zur Muhlen C, Fink Petri A, Salaklang J, et al. Imaging monocytes with iron oxide nanoparticles targeted towards the monocyte integrin MAC-1 (CD11b/CD18) does not result in improved atherosclerotic plaque detection by in vivo MRI. Contrast Media Mol Imaging. 2010;5:268-275. doi: 10.1002/ cmmi.384.

58. Tavakoli S, Vashist A, Sadeghi MM. Molecular Imaging of Plaque Vunerability. J Nuc Cardiol. 2014;21:1112-1128. doi: 10.1007/s12350-014-9959-4. 